\title{
BMJ Open Efficacy and neural mechanism of acupuncture treatment in older adults with subjective cognitive decline: study protocol for a randomised controlled clinical trial
}

Chao-Qun Yan, ${ }^{1,2}$ Ping Zhou, ${ }^{1}$ Xu Wang, ${ }^{3}$ Jian Feng Tu, ${ }^{1}$ Shang-Qing Hu, ${ }^{1}$ Jian-Wei Huo, ${ }^{4}$ Zhong-Yan Wang, ${ }^{4}$ Guang-Xia Shi, ${ }^{1}$ Ya-Nan Zhang, ${ }^{4}$ Jun-Qiu Li, ${ }^{4}$ Jun Wang, ${ }^{2}$ Cun-Zhi Liu (D) ${ }^{1}$

\section{ABSTRACT}

Introduction Subjective cognitive decline (SCD) refers to individuals' perceived decline in memory and/or other cognitive abilities relative to their previous level of performance, while objective neuropsychological deficits are not observed. SCD may represent a preclinical phase of Alzheimer's disease. At this very early stage of decline, intervention could slow the rate of incipient decline to prolong and preserve cognitive and functional abilities. However, there is no effective treatment recommended for individuals with SCD. Acupuncture, as a nonpharmacological intervention, has been widely employed for patients with cognitive disorders.

Methods and analysis The proposed study is a randomised, assessor-blinded and placebo-controlled study that investigates the efficacy and mechanism of acupuncture in SCD. Sixty patients with SCD will be randomly allocated either into an acupuncture group or a sham acupuncture group. They will receive 24 sessions of real acupuncture treatment or identical treatment sessions using a placebo needle. Global cognitive changes based on a multidomain neuropsychological test battery will be evaluated to detect the clinical efficacy of acupuncture treatment at baseline and end of treatment. MRI scans will be used to explore acupuncture-related neuroplasticity changes. Correlation analyses will be performed to investigate the relationships between the changes in brain function and symptom improvement.

Ethics and dissemination The trial was approved by the research ethics committee. The results of the study will be published in a peer-reviewed academic journal and will also be disseminated electronically through conference presentations.

Trial registration number NCT03444896.

employer(s)) 2019. Re-use permitted under CC BY-NC. No commercial re-use. See rights and permissions. Published by BMJ.

For numbered affiliations see end of article.

Correspondence to Professor Cun-Zhi Liu; Icz_tg@126.com

\section{INTRODUCTION}

Dementia is the greatest global challenge for health and social care in the 21 st century. ${ }^{1}$ Globally, about 47 million people were living with dementia in 2015, and this number is projected to triple by 2050 , based on the
Strengths and limitations of this study

- Compared with the neuropsychological tests alone as efficacy evaluations, the combination with in vivo measures of brain alterations in this study will be more sensitive in detecting acupuncture efficacy.

- This study will first uncover the efficacy and neural mechanism of acupuncture treatment in older adults with subjective cognitive decline.

- A multidomain neuropsychological test battery will be employed, which can assess multiple cognitive domains including executive function, attention, visuospatial function and language.

- Sham needles and adhesive pads will be used for better patient blinding.

- A potential limitation is that not a large sample size will be performed in this study.

World Alzheimer Report (2015). Alzheimer's disease $(\mathrm{AD})$ is the most common form of dementia. ${ }^{2}$ Currently, there is no effective cure for $\mathrm{AD}$, and the available treatments have only moderately alleviated the symptoms. ${ }^{3}$ Therefore, prevention is essential to reduce the dementia epidemic. ${ }^{45}$ The long "preclinical' phase of $\mathrm{AD}$ provides an opportunity for individuals to participate in treatment trials to delay or prevent cognitive decline. ${ }^{6-8}$

Subjective cognitive decline (SCD) usually occurs in older adults, and it refers to the self-perception of cognitive decline, when individuals perform cognitive tests within normal limits and have preserved activities of daily living. ${ }^{9}$ A review demonstrated that the prevalence of these complaints in persons aged 65 years or more varied from $25 \%$ to $50 \%{ }^{10}$ Actually, many older adults claim cognitive decline might be regarded as hypochondriacs by professional healthy carers. 
However, several lines of evidence from longitudinal ageing studies suggest that older adults with SCD are more likely to present $\mathrm{AD}$ biomarkers than their healthy peers. About $60 \%$ of SCD individuals will decline to mild cognitive impairment (MCI) and AD over a 15 -year period. ${ }^{11} 12$ People with SCD have more brain abnormalities such as hippocampal volume loss and hypometabolism compared with healthy controls. ${ }^{13}$ Moreover, cerebrospinal amyloid $\beta$ is strongly predictive of subsequent clinical progression in patients with SCD. ${ }^{14}$ This suggests that, for some older adults, SCD may represent a preclinical phase of AD.

There is still much plasticity in SCD. The patients in this stage may not progress to dementia or even revert to no cognitive impairment after effective treatment. Development of safe and effective interventions in early $\mathrm{AD}$ stages is important. Specific criteria to define SCD have been published, ${ }^{915}$ but clinical trials in SCD are in their infancy, and no pharmacological treatment or intervention is currently recommended for individuals with SCD. Despite these difficulties, the development of new treatments should be encouraged. ${ }^{16}$

The accumulated evidence suggests that non-pharmacological intervention may benefit cognitive function in older adults with SCD. ${ }^{17}$ Acupuncture, as a non-pharmacological intervention, has been widely used for patients with cognitive disorders. The WHO reports that acupuncture treatment can be beneficial for vascular dementia. A number of clinical studies have provided evidence that acupuncture is beneficial for the treatment of dementia or MCI. ${ }^{18}$ A systematic review supports the use of acupuncture for MCI. ${ }^{19}$ Animal studies showed that acupuncture elicits its effects by mediation of neural plasticity in pathological conditions. ${ }^{20}$ However, the efficacy of acupuncture in patients with SCD has not be investigated. An experimental study is needed to test this hypothesis.

By definition, individuals with SCD are within the normal range on clinical-neuropsychological tests. It will be difficult to detect response in this population compared with people who already have clinically manifested impairment (eg, MCI) due to a ceiling effect. As such, neuropsychological tests in conjunction with in vivo measures of brain function such as electroencephalography, or functional MRI (fMRI), may be a more sensitive alternative to neuropsychological tests. ${ }^{21-23}$ Brain alterations are subtle but measurable to identify individuals at risk for $\mathrm{AD}$ well before cognitive symptoms are manifested by using fMRI, allowing researchers to use neuroimaging to ascertain response after intervention sensitivity. ${ }^{24}$ For better ascertaining the clinical response and unveiling the mechanism, brain alterations measured by fMRI could also be used to evaluate the efficacy of acupuncture.

\section{OBJECTIVES}

This study is a randomised controlled functional brain imaging trial with 12 weeks of treatment, aiming at: (1) evaluating the effects of acupuncture treatment on

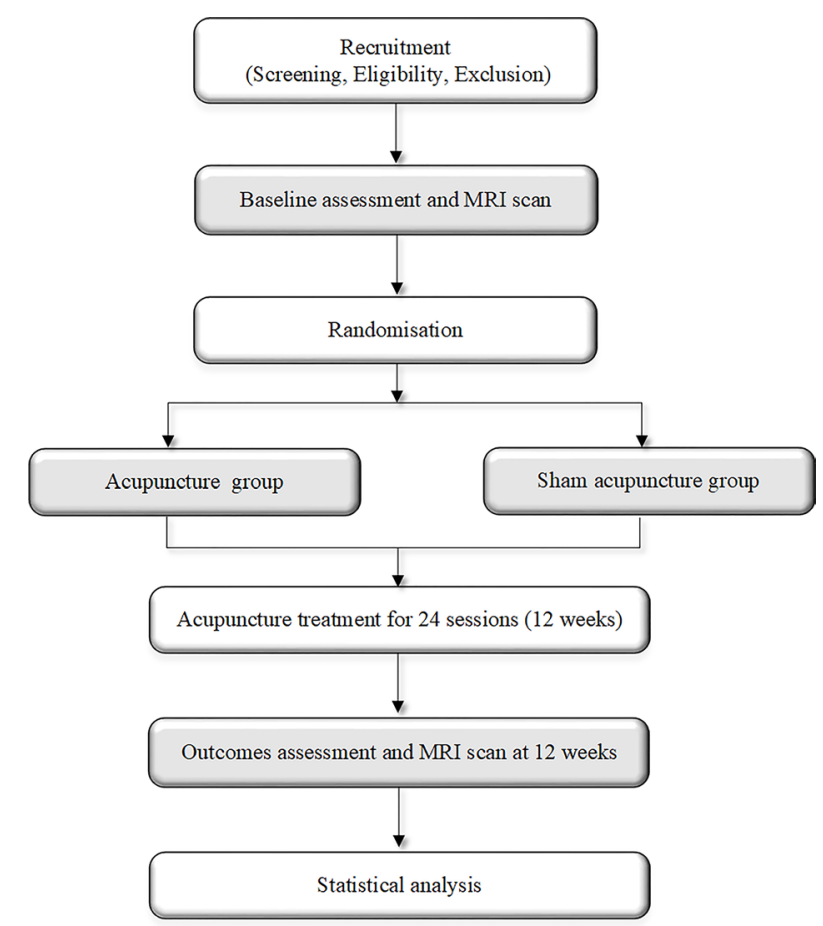

Figure 1 Flow chart.

cognitive function in older adults with SCD; (2) exploring the central mechanism of the long-lasting effect of acupuncture on SCD; and (3) investigating the safety of acupuncture treatment on SCD.

\section{METHODS}

Design

The Consolidated Standards of Reporting Trials (CONSORT) statement for non-pharmacological interventions has been used as a framework for development of the methodology for this project. ${ }^{25} 26$

This trial is a 1:1 randomised single-blinded and placebo-controlled study with two parallel groups involving elderly adults. The study is designed to examine the effect and neural mechanism of acupuncture treatment on cognitive function before and after a 12-week intervention period. As shown in figure 1, the cognitive assessments and MRI scans will be performed at baseline and immediately after the completion of the intervention.

\section{Participants}

The study will mainly take place in Dongcheng, Fengtai and Shunyi districts in Beijing. People with SCD will be recruited from community-dwelling population in those districts. In order to have better insight into the trial for community residents, science popularisation lectures about dementia and advertisement of the trial will be given in community service centres. The brochures and posters will also be distributed. Besides, advertisement of the trial will be shared in the official account on WeChat (China's most popular social media platform) of the Beijing Hospital of Traditional 
Chinese Medicine Affiliated to Capital Medical University and health website (www.39.net) or local newspaper for recruiting participants. Interested individuals will be screened in the clinic, community service centres or by phone using the inclusion/exclusion criteria. All those who meet the inclusion criteria will receive a study information sheet including the design, procedure, benefits and risks of the study. Before the study procedure started, the subjects will have to provide signed written informed consent forms. The diagnosis of SCD will be based on the published SCD research criteria proposed by the Subjective Cognitive Decline Initiative. ${ }^{9}$ In this study, the patients who answer 'yes' to the question 'Do you have problem in memory?' in the initial screening by phone or face-to-face interviews will be selected to the next step. Besides, the Subjective Cognitive Decline Questionnaire 9 (SCDQ9) is further used for SCD screening. Only the patients with the SCD-Q9 score more than 5.0 will pass the screening.

\section{Inclusion criteria}

- Male and female adults aged 55-75.

- Native Chinese speakers who are right handed and have at least a primary school education.

- Self-reported persistent memory decline compared with a previous normal status within the last 5 years, which is confirmed by caregivers.

- Normal age and education-adjusted performance on neuropsychological test including the Chinese version of Mini-Mental State Examination, ${ }^{27}$ the Auditory Verbal Learning Test (AVLT; the short-term delayed free recall, the long-term delayed free recall and the recognition test), ${ }^{28}$ Trail Making Test $(\mathrm{TMT})^{29}$ and Animal Fluency Test (AFT). ${ }^{30}$

- No or minimal impairment in activities of daily living.

\section{Exclusion criteria}

- Presence of a positive neurological history (eg, traumatic brain injury, stroke, Parkinson's disease, multiple sclerosis).

- Treatments that would affect cognitive function (eg, treatment for an acute psychiatric episode, therapy with memantine, rivastigmine and donepezil).

- Presence of significant psychiatric history (eg, bipolar disorder, schizophrenia) and/or severe anxiety and depression.

- Presence of serious heart, kidney, liver, gastrointestinal, infectious, endocrine disease or cancer.

- History of alcohol or drug abuse/addiction.

- Any contraindications for MRI scans (eg, aneurysm clip pacemaker).

- Significant visual and/or auditory impairment uncorrected by aids, and unable to perform neuropsychological evaluations.

- Currently enrolled in another research study.

- Received acupuncture treatment in the preceding month.
During the trial period, patients with SCD who meet the following criteria will be excluded from the study:

- Taking medication or receiving additional treatment that is expected to affect the cognitive function (eg, tranquillisers, antianxiolytics, hypnotics, nootropics and cholinomimetic agents).

- Withdrawal of consent for study participation because the patient does not wish to continue.

- Missing more than 5 of 24 acupuncture treatment sessions.

- Occurrence of a serious adverse event that the doctors consider the treatment should be termination.

- Critical protocol violation such as violation of eligibility criteria.

\section{Intervention}

Patients will receive 24 acupuncture treatment sessions over 12 weeks (twice a week). Hwato brand single-use acupuncture needles (size $0.35 \times 25 \mathrm{~mm}$ or $0.35 \times 40 \mathrm{~mm}$ ), pragmatic placebo needles (size $0.30 \times 25 \mathrm{~mm}$ ) and SDZ-V electroacupuncture apparatuses will be used.

For the acupuncture group (figure 2), acupuncture needles will be placed at acupoints Baihui (DU20), Shengting (DU24), Fengfu (DU16), Fengchi (GB20), Danzhong (RN17), Zhongwan (RN12), Qihai (RN6), Neiguan (PC6), Tongli (HT5), Xuehai (SP10), Zusanli (ST36), Zhaohai (KI6), Xinshu (BL15) and Yixi (BL45). After skin disinfection in patients in the supine position, the adhesive pads are pasted on the acupoints surface except for Baihui (DU20) and Shengting (DU24). Then, the acupuncture needles will be inserted through the adhesive pads into the skin depending on the location of the needle. The locations are shown in table 1 . Manual acupuncture by acupuncturists using small, equal manipulations of twirling, lifting and thrusting will be performed on all needles to reach deqi. The patients will feel the deqi sensation, such as soreness, numbness, distention, heaviness and other sensations. Paired electrodes from the electroacupuncture apparatus will be attached to the needle holders of the DU20 and DU24. A dilatational wave of $2-100 \mathrm{~Hz}$ and a current intensity of 1-5 mA will be performed according to the degree of needle handle shivering, ignoring the patients' feelings. The needles will be extracted after $20 \mathrm{~min}$ for each treatment. The acupoints of DU16, BL15 and BL45 will achieve deqi in patients in a sitting position without retaining the needle.

For the sham acupuncture group, sham acupoints at locations away from known acupuncture points will be used to minimise physiological effects, and the locations of sham acupoints are shown in table 2. Patients will receive non-insertive acupuncture using the pragmatic placebo needles (Hwato brand, size $0.35 \times 25 \mathrm{~mm}$; online supplementary figure 1). Similar to the acupuncture group, the adhesive pads are initially pasted on the sham acupoints surface except for sham acupoint 1 . The pragmatic placebo needles with a blunt tip will be placed on the adhesive pads. In order to minimise the physiological 


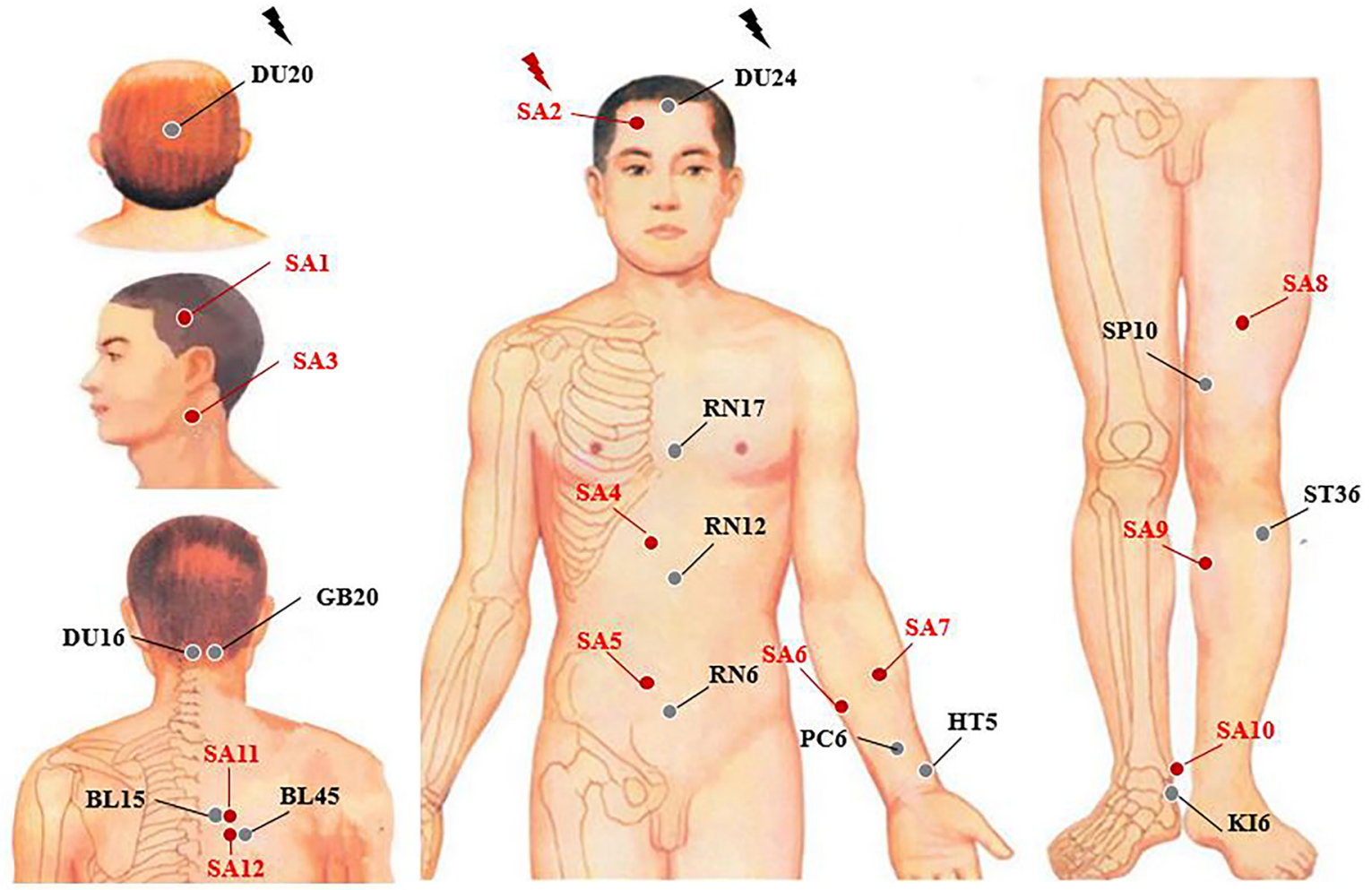

Figure 2 Location of acupoints or sham acupoints in the trial. BL15, Xinshu; BL45, Yixi; DU16, Fengfu; DU20, Baihui; DU24, Shengting; GB20, Fengchi; HT5, Tongli; KI6, Zhaohai; PC6, Neiguan; RN6, Qihai; RN12, Zhongwan; RN17, Danzhong; SA, sham acupoint; SP10, Xuehai; ST36, Zusanli.

effect, acupuncturists will be instructed to lightly place the sham needles with a blunt tip on the adhesive pads with no manipulation. The acupuncture needle will be inserted to a shallow depth at the sham acupoint 1 , which does not penetrate below the skin, and needle manipulation for deqi. Sham acupoint 11 and sham acupoint 12 will be inserted with sham needles without retaining the needle. Paired electrodes will be attached to the needle holders of the bilateral sham acupoint 2 but with no electricity output.

\section{MRI protocol}

Patients will undergo brain MRI at baseline and after treatments. The MRI scan will be performed with a 3.0 Tesla superconductor (Skyra, Siemens, Erlangen, Germany) in the Beijing Hospital of Traditional Chinese Medicine Affiliated to Capital Medical University. The parameters of sequences employed in this study are provided by China Association of Brain Imaging ( www.abimaging.org). Sagittal structural images will be acquired using a magnetisation prepared rapid gradient-echo three-dimensional (3D) T1-weighted sequence with the following parameters: repetition time (TR)/ echo time $(\mathrm{TE})=2530 / 2.98 \mathrm{~ms}$, flip angle $=7^{\circ}$, inversion time $=1100 \mathrm{~ms}$, matrix $=256 \times 256,1 \mathrm{~mm}$ slice thickness without slice gap. T2*-weighted functional images will be collected using a gradient-echo echo-planar imaging (EPI) sequence with the following parameters: TR=3200 $\mathrm{ms}, \mathrm{TE}=407 \mathrm{~ms}$, flip angle $=120^{\circ}$.
Resting state fMRI and task fMRI will be performed using an EPI sequence with the following parameters: TR/ $\mathrm{TE}=2000 / 30 \mathrm{~ms}$, flip angle $=90^{\circ}$, matrix $=64 \times 64$, slice thickness $=3.5 \mathrm{~mm}$ with $1 \mathrm{~mm}$ slice gap. Diffusion tensor imaging (DTI) will use a double spin-echo EPI sequence (TR/ $\mathrm{TE}=12000 / 77 \mathrm{~ms}$, flip angle $=90^{\circ}$, volume interval $=12$, $2 \mathrm{~mm}$ thick axial slices). Arterial spin labelling (ASL) imaging of the whole brain is performed by use of a 3D pseudocontinuous ASL sequence (TR/TE $=5000 / 15.92$ ms, flip angle $=180^{\circ}$, slice thickness $=3.5 \mathrm{~mm}$; labelling duration, number of slices $=40$ ).

All scans will be reviewed qualitatively by two radiologists to screen for possible brain lesions or structural abnormalities. DTI images will be analysed using the PANDA package. ${ }^{31}$ fMRI data were collected during the memory task and at rest. ASL data will be performed with the resting state fMRI (DPARSF) toolbox and SPM8 (http:// www.fil.ion.ucl.ac.uk/spm/software/spm8) for MATLAB. Brain activation, connectivity changes and cerebral blood flow will be compared between the two groups before and after treatment.

\section{Outcome measures}

Clinical outcome assessments

Global cognitive function based on a composite score will be used to evaluate the clinical efficacy of acupuncture treatment at baseline, and at the end of the 12-week treatment period. It will be computed by averaging z scores from a multidomain neuropsychological test battery that 
Table 1 Location of acupoints used in the acupuncture group

\begin{tabular}{|c|c|c|}
\hline Acupoints & Location & Depth \\
\hline Baihui (DU20) & $\begin{array}{l}5 \text { cun directly above the midpoint of the posterior hairline, or at the midpoint of the } \\
\text { line connecting the apexes of the two auricles. }\end{array}$ & 0.2 cun \\
\hline Fengfu (DU16) & $\begin{array}{l}\text { On the back of neck, } 1 \text { cun directly above the midpoint of the posterior hairline, } \\
\text { directly below the external occipital protuberance. }\end{array}$ & $0.5-0.1$ cun \\
\hline Fengchi (GB20) & $\begin{array}{l}\text { On the nape, below the occiput, at the level of Fengfu, in the depression between the } \\
\text { upper portion of sternocleidomastoideus and trapezius muscle. }\end{array}$ & $0.5-0.8$ cun \\
\hline Danzhong (RN17) & $\begin{array}{l}\text { On the anterior median line of the chest, at the level of the fourth intercostal space, at } \\
\text { the midpoint between the two nipples. }\end{array}$ & 0.5 cun \\
\hline Zhongwan (RN12) & On the anterior median line of the upper abdomen, 4 cun above the umbilicus. & $1-1.5$ cun \\
\hline Qihai (RN6) & On the anterior median line of the lower abdomen, 1.5 cun below the umbilicus. & $1-1.5$ cun \\
\hline Neiguan (PC6) & $\begin{array}{l}\text { On the palmar aspect of forearm, } 2.0 \text { cun above the transverse crease of the wrist, } \\
\text { between the tendons of palmaris longus and flexor carpi radialis muscle. }\end{array}$ & $0.5-0.1$ cun \\
\hline Xuehai (SP10) & $\begin{array}{l}\text { When the knee is flexed, on the medial aspect of the thigh, the point is } 2 \text { cun above } \\
\text { the mediosuperior border of the patella, on the bulge of the medial portion of muscle } \\
\text { quadriceps femoris. }\end{array}$ & $0.8-1$ cun \\
\hline Zusanli (ST36) & $\begin{array}{l}3 \text { cun directly below Dubi* and } 1 \text { fingerbreadth lateral to the anterior border of the } \\
\text { tibia. }\end{array}$ & $0.8-1$ cun \\
\hline Zhaohai (KI6) & On the depression below the tip of the medial malleolus. & $0.5-0.8$ cun \\
\hline Xinshu (BL15) & 1.5 cun from the lower border of the spinous process of the fifth thoracic vertebra. & $0.3-0.5$ cun \\
\hline Yixi (BL45) & 3 cun from the lower border of the spinous process of the sixth thoracic vertebra. & $0.5-0.8$ cun \\
\hline
\end{tabular}

*Dubi location: when the knee is flexed, the point is at the knee, below the patella, in the depression from the patella ligament.

includes eight tests. AFT assesses the language and executive function by examining categorical verbal fluency. ${ }^{30}$ TMT part A and B examines graphomotor speed, attention and executive function. ${ }^{32}$ Digit Symbol Substitution Test is used to assess processing speed, attention and concentration. ${ }^{33}$ Clock Drawing Test can assess multiple cognitive functions, including verbal understanding, memory, abstract thinking and executive functions. ${ }^{34}$
Digit Span Test is used to measure working memory's number storage capacity. Stroop Color and Word Test is a classic instrument for the assessment of selective attention, cognitive flexibility, cognitive inhibition and information processing speed. ${ }^{35}$ AVLT is a powerful neuropsychological test that assesses episodic memory. ${ }^{36} 37$

All patients will fill out an SCD- $Q^{38}$ to assess their degree of SCD. The Face Recognition Questionnaire

Table 2 Location of sham acupoints used in the sham acupuncture group

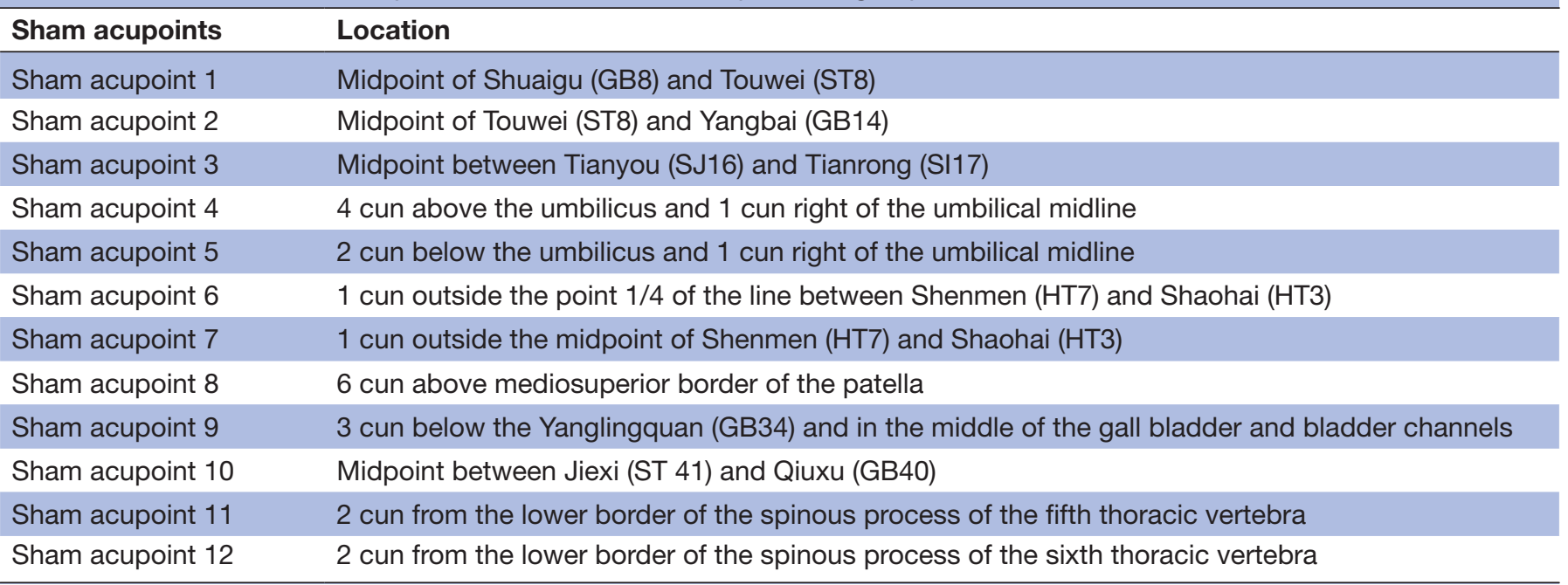


Pre-scan session

MRI scan session: total session time $=\mathbf{4 4 . 4} \mathbf{m i n}$
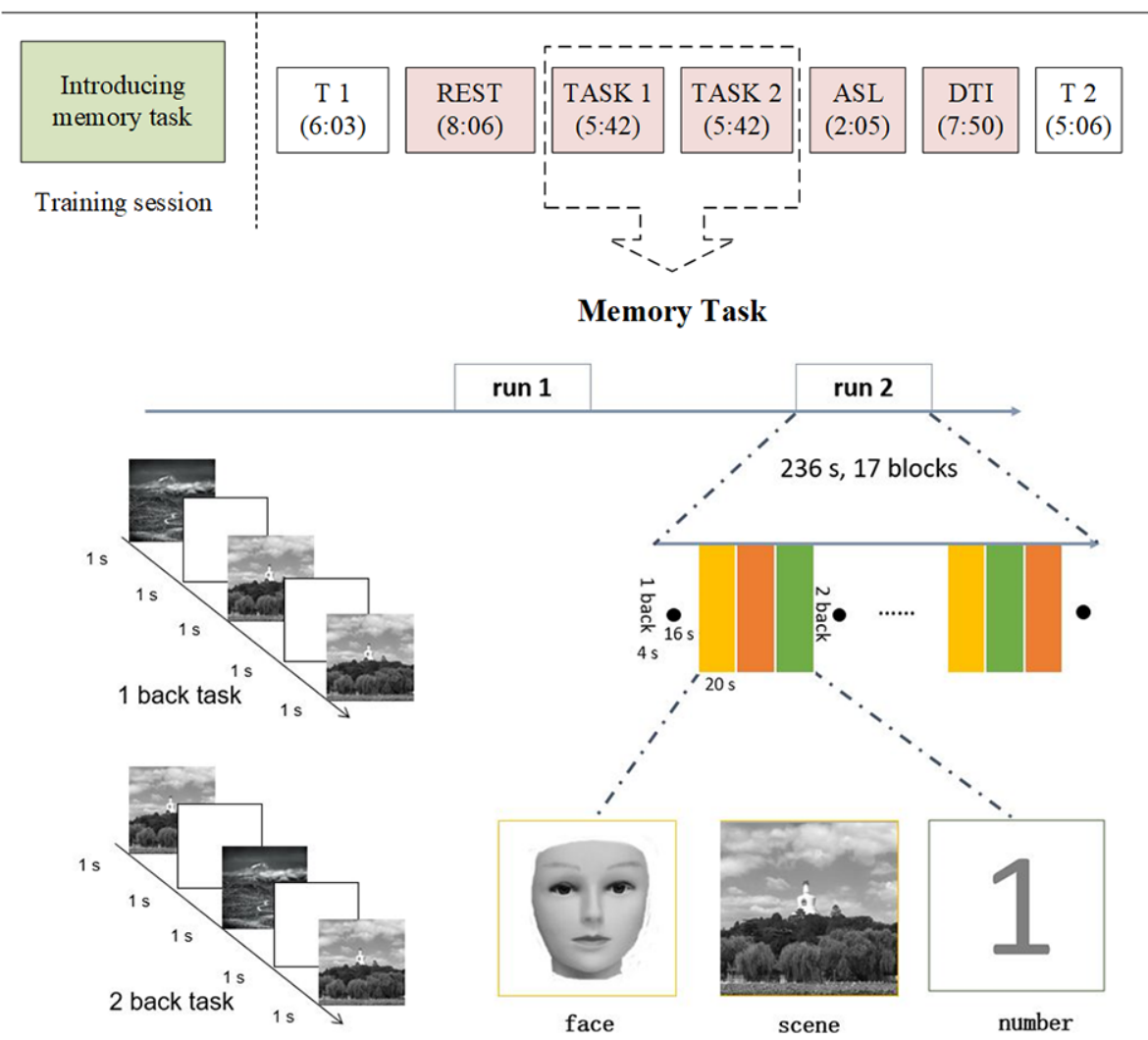

Figure $3 \mathrm{MRI}$ experimental paradigm and an illustrative diagram of the memory task. ASL, arterial spin labelling; DTI, diffusion tensor imaging.

and the Santa Barbara Sense of Direction Scale ${ }^{39}$ will be used to measure the ability for facial recognition and direction. Depressive symptoms and sleep quality will be measured by Geriatric Depression Scale and Pittsburgh Sleep Quality Index. ${ }^{40}$

\section{Neuroplasticity assessments}

In this study, neuroplasticity changes between the two groups will be measured by fMRI. Before the acupuncture treatment, patients will complete the fMRI scan within 3 days. They will also have the follow-up fMRI scan within 3 days after the completion of their intervention (figure 3). Brain activity and functional connectivity will be assessed under a resting state and a memory task. Group difference on cerebral blood flow and white matter integrity will be assessed by means of ASL or DTI.

\section{Memory task}

The patients will be trained before entering the fMRI scanner. They will complete a practice version of the memory task paradigm in computer. This practice mock test will show the correct number, and they need to perform the tasks with an accuracy criterion of $80 \%$ to ensure that the patients understand how to do the memory task in the scanner. A blocked periodic design that incorporated alternating 1-back and 2-back tasks will be used. Patients will view facial pictures, scene pictures or single digits (0-9, black on a white background). In the 1-back task, the patients are required to press a button when a facial picture, scene picture or a digit appears the same as the one before. In the 2-back task, the patients are asked to press a response button if the current facial picture, scene picture or the digit is the same as the one which was presented two trials before. The stimuli consist of 17 blocks for $236 \mathrm{~s}$ and are presented on a computer using MATLAB V.8.6 (MathWorks, Natick, MA, USA) for $1 \mathrm{~s}$, with an interstimulus interval of $1 \mathrm{~s}$. Trial types within blocks are presented in pseudorandomised order.

Once in the scanner, anatomical and functional scans will be performed. During the scanning, all patients are asked to lie quietly in the scanner with their eyes open, trying to avoid thinking systematically, and moving as little as possible. In resting state fMRI scanning, the patients are instructed to view centrally placed fixation cross $(+)$, and try to not think of anything. Two memory task runs will be conducted after $\mathrm{T} 1$ and resting state scans.

\section{Apolipoprotein E genotyping}

A TIANamp Blood DNA Kit (Tiangen Biotech, Beijing, China) will be used to isolate high-molecular-weight DNA. The genotype of apolipoprotein E (APOE) will be detected by APOE Gene Detection Kit (Wuhan YZY Biopharma, Wuhan, China) with E2 and E4 reaction liquid. Genomic DNA and reaction liquid will be placed on a 7500 HT Fast Real-Time PCR (Applied Biosystems, Foster City, USA) 
with the following conditions: uracil- $\mathrm{N}$-glycosylase treatment at $37^{\circ} \mathrm{C}$ for $10 \mathrm{~min}$, denaturation at $95^{\circ} \mathrm{C}$ for $5 \mathrm{~min}$, followed by 40 cycles at $95^{\circ} \mathrm{C}$ for $15 \mathrm{~s}$ and annealing at $60^{\circ} \mathrm{C}$ for $60 \mathrm{~s}$ (acquiring the fluorescence signal in this step). According to the amplification plot, the genotype of APOE will be determined.

\section{Safety and monitoring}

Treatment-related adverse events, including haematoma, bleeding, subcutaneous haemorrhage, serious pain, local infection and fatigue, will be compared among patients in the acupuncture group and the sham acupuncture group, and will be documented at each treatment session. In order to ensure consistency, the neuropsychological tests and other assessment scales will be performed by the same researcher at baseline and the end of treatment. A professional staff will check the imaging data for quality and protocol conformity after each scanning session. Patients will undergo blood routine test and blood biochemical tests including blood glucose, homocysteine, total cholesterol, and so on, before randomisation and at the end of treatment.

\section{Sample size and blinding}

Task fMRI studies characteristically include small sample size $^{41}$ and thus have a low statistical power. The statistically underpowered study by definition means that a study will have less of a chance for detecting significant effects. ${ }^{42}$ Therefore, power-based sample size will be calculated prior to fMRI data collection. ${ }^{43}$ Fortunately, approaches for sample size calculations in studies using fMRI have been developed.

By using the non-central random field theory, Hayasaka et al estimated that at least 12 subjects would be required to detect signals in either of the auditory cortices with at least $80 \%$ power. ${ }^{44}$ They also found that approximately 13 subjects would be required to detect signals in the auditory cortices with $80 \%$ power, when generated a sample size map based on the mock pilot analysis. ${ }^{44}$ Adopting a simulation-based method to calculate statistical power for group-level fMRI studies, Desmond and Glover found a minimum of 12 subjects are required to achieve $80 \%$ power at $\alpha=0.05$ at the single voxel level. ${ }^{45}$ For a more realistic threshold, twice as many subjects are recommended to maintain this level of power after correcting for multiple comparisons. For a stricter alpha of 0.000002 , approximately 25 subjects are needed. Mumford and Nichols recommended 20 subjects and a type I error of $\alpha=0.005$ should probably be used, and this power calculation is based on a non-central T or F distribution. ${ }^{46} \mathrm{We}$ used the largest sample size of 25 for each group with an estimated dropout rate and loss of data due to head motion, then we planned to enrol 60 participants in the two groups.

Eligible patients will be randomly assigned into either the acupuncture or sham acupuncture group after signing written informed consent forms via a randomisation digital table with a 1:1 ratio. Blocked randomisation

\begin{tabular}{|l|c|c|c|c|}
\hline \multicolumn{5}{|c|}{ Study period } \\
\hline Items & \multicolumn{2}{|c|}{ Baseline } & Treatment phase & Outcome assessment \\
\hline Time point & -1 week & 0 week & $1-12$ weeks & 13 -14 weeks \\
\hline Enrolment & & & & \\
\hline Eligibility screen & $\mathrm{X}$ & & & \\
\hline Informed consent & $\mathrm{X}$ & & & \\
\hline Examination & & $\mathrm{X}$ & & \\
\hline Randomisation & & $\mathrm{X}$ & & \\
\hline Intervention & & & & \\
\hline Acupuncture (n=30) & & & $\mathrm{X}$ & \\
\hline Sham acupuncture $(\mathbf{n}=30)$ & & & $\mathrm{X}$ & \\
\hline MRI scan & & & & \\
\hline Acupuncture & & $\mathrm{X}$ & & $\mathrm{X}$ \\
\hline Sham acupuncture & & $\mathrm{X}$ & & $\mathrm{X}$ \\
\hline Assessment & & & & $\mathrm{X}$ \\
\hline AFT & & $\mathrm{X}$ & & $\mathrm{X}$ \\
\hline TMT & & $\mathrm{X}$ & & $\mathrm{X}$ \\
\hline DSST & & $\mathrm{X}$ & & $\mathrm{X}$ \\
\hline SCWT & & $\mathrm{X}$ & & $\mathrm{X}$ \\
\hline AVLT & & $\mathrm{X}$ & & $\mathrm{X}$ \\
\hline SCDQ & & $\mathrm{X}$ & & $\mathrm{X}$ \\
\hline SBSDS & & $\mathrm{X}$ & & $\mathrm{X}$ \\
\hline HAMD & & $\mathrm{X}$ & & $\mathrm{X}$ \\
\hline HAMA & & $\mathrm{X}$ & & $\mathrm{X}$ \\
\hline PSQI & & $\mathrm{X}$ & & $\mathrm{X}$ \\
\hline Safety & & & & \\
\hline Laboratory test & & $\mathrm{X}$ & & $\mathrm{X}$ \\
\hline Adverse events & & & & \\
\hline & & & & \\
\hline
\end{tabular}

Figure 4 Standard Protocol Items: Recommendations for Interventional Trials (SPIRIT) and the schedule of the trial. AFT, Animal Fluency Test; AVLT, Auditory Verbal Learning Test; DSST, Digit Symbol Substitution Test; SBSDS,Santa Barbara Sense of Direction Scale; HAMD, Hamilton Rating Scale forDepression; HAMA, Hamilton Rating Scale for Anxiety; PSQI, Pittsburgh Sleep Quality Index; SCDQ, Subjective Cognitive Decline Questionnaire; SCWT, Stroop Color and Word Test; TMT, Trail Making Test.

with a block size of 6 will be employed to ensure balance within the two groups. The randomisation sequence will be generated by a third-party professional statistician using computer-generated randomisation digital table by using SAS V.9.2 (SAS Institute). The randomisation list will be stored by a non-involved investigator and out of reach and sight of the involved investigators. The allocation schedule will be using a telephone randomisation procedure. The randomisation list was restricted to this research coordinator and was concealed from other study personnel. The patients, outcome assessors and statisticians will be blinded to treatment allocation. Patients are told that they will receive one of two effective interventions randomised after enrolment. During the acupuncture treatment, the adhesive pads are pasted on the acupoints or sham acupoints after skin disinfection. The true or sham needles with a blunt tip will be placed in the adhesive pads. Patients in different groups will be assigned into separate cubicles to refrain from communication.

\section{Data collection and management}

At baseline, information of patients about age, sex, education, dominant hand and medical history will be collected (figure 4). For all patients who met the inclusion criteria, the neuropsychological tests will be performed before and after treatment in a quiet room by evaluators, who have been trained by the organisation of Alliance of early AD. All of the MRI scans will be conducted in the same machine, and the operators have been technically trained by a professional engineer from the China Association of 
Brain Imaging. Blood samples will be collected in the morning and handled by two persons. After each treatment session, adverse events will be recorded in a participant's diary card by acupuncturists.

A case report form (CRF) will be used to collect the clinical data for each patient, labelled by unique numeric identifier and recorded by a trained graduate student. The data in the CRF will be verified for accuracy, missing data and data consistency by a clinical research associate. Paper-based research data will be entered into an EpiData electronic database. For reducing errors in data entry, data will be entered independently twice by two independent people, and value pairs will then be compared for discordances, followed by resolution of discordances by referral to the original data source. The EpiData will export data in a Microsoft Excel spreadsheet format. The data will be entered in an electronic statistical package SPSS software (SPSS V.12.0 KO for Windows) for ease of statistical analysis.

\section{Statistical analysis}

Clinical data analysis

In this study, intent-to-treat analysis and per-protocol analysis will be used. In this study, the intent-to-treat population consisted of all randomised patients who received at least one dose of treatment and had a complete baseline assessment. The per-protocol population defined as all randomised patients who did not discontinue prematurely completed the 12 weeks' treatment and had MRI scans before and after treatment. Observation of histograms and normal probability plots and a Shapiro-Wilk test will be performed to determine whether the data followed a normal distribution. Distributed data will be described using means, SDs and 95\% CIs. Clinical outcome analyses will be done with SPSS software (SPSS V.22.0 KO for Windows), with a significance level of 0.05 , and all hypothesis tests are two tailed.

Baseline demographic characteristics between the two groups will be analysed with $\chi^{2}$ test or Fisher's exact test for categorical measures, and with the t-test or Wilcoxon rank-sum test for continuous measures. The clinical outcome of the global cognitive function based on composite scores will be compared between the acupuncture group and the sham acupuncture group. The composite scores will be created by converting all individual cognitive scores to standardised $\mathrm{z}$ scores. As previously described, ${ }^{47}$ we computed $\mathrm{z}$ scores by subtracting the baseline group mean and dividing the baseline group SD, and then averaging the standardised $\mathrm{z}$ scores across all tests. The general linear model will be used to examine change in cognitive scores. For the other outcomes, if it agreed with normal distribution, an independent t-test will be used. Otherwise, for abnormal distribution, the data will be analysed with Wilcoxon test. The statisticians who analysed the data are blinded to the test settings.
MRI data analysis

For imaging, data will be analysed using DPABI toolkit performing on MATLAB V.8.6 (MathWorks) to detect any changes in brain function due to acupuncture treatment. After data preprocessing, some graph theory-based or data-driven approaches will be performed to investigate neuroplasticity between the two groups, such as regional homogeneity, amplitude of the low-frequency fluctuation and voxel-wise degree centrality. A two-sample t-test will be conducted to investigate the differences in brain regions between the acupuncture group and the sham acupuncture group in the DPARSF software. Multiple comparisons will be used in order to better control for a highly inflated false positivity rate. Pearson's correlation analysis will be performed to examine the association between the fMRI image data and clinical variables.

\section{Ethics and dissemination}

The CONSORT statement and the CONSORT statement for non-pharmacological interventions have been used as a framework for development of the methodology for this project. $^{2425}$

If any modifications or decision are made, amendments will be reviewed and approved by the ethics committee, and new protocols would be uploaded to ClinicalTrials. gov. The results of the study will be published in a peer-reviewed academic journal and will also be disseminated electronically through conference presentations.

\section{Patient and public involvement}

Currently, this trial is at the recruitment phase with no patient involved in. The participants will be able to view the study results via social media.

\section{DISCUSSION}

$\mathrm{SCD}$ may be the initial phase of $\mathrm{AD}$, and could serve as a window of opportunity for interventions at an early disease stage. ${ }^{23}$ This study is planned as a randomised, assessor-blinded, placebo-controlled trial to evaluate the efficacy and central mechanism of acupuncture treatment on SCD compared with a sham acupuncture group. To our knowledge, this trial is also the first study to investigate the effect of acupuncture in patients with SCD.

This study has several strengths. First, we will assess patients with SCD on multiple levels, including multidomain neuropsychological tests, functional brain alterations, cerebrovascular risk factors and APOE genotyping. This multidomain assessment will be used to identify possible biomarkers involved in the effects of acupuncture in SCD.

Second, the neuropsychological assessment in the present study is a multidomain neuropsychological test battery that included eight tests, which can assess multiple cognitive domains including executive functions, attention, visuospatial functioning and language. Memory decline associated with AD risk factors, ${ }^{49} 49$ and then most instruments were used previously to measure SCD focus 
on memory. ${ }^{50}$ However, it might be too restrictive to limit the SCD assessment to memory, in particular for atypical forms of $\mathrm{AD} .{ }^{951}$ In this study, multidomain cognitive function will be assessed.

Additionally, functional brain alterations will be used as outcome measures. Compared with the neuropsychological tests alone, the combination of in vivo measures of brain alterations in this study will be more sensitive in detecting acupuncture efficacy. A smaller training effect and ceiling effect may be found when using MRI. ${ }^{52}$ Furthermore, it can be used as a tool to understand the mechanism of the acupuncture's effects. fMRI can identify whether neural efficiency is improved or the brain connectome is reorganised to achieve cognitive enhancement. ${ }^{53}$

A potential limitation is that the patients in this study will be recruited from the community. Considering the research environment, individuals recruited from a memory clinic may have a higher probability of having preclinical AD. They had specific concerns sufficient to prompt a medical visit. ${ }^{15}$ However, community-based studies have shown some predictive value of SCD for cognitive decline and dementia. ${ }^{10} 54$ Another potential limitation of our study is that it will not include a large sample size, and this may lead to the need for a future study. The main feature of our study is to systemically investigate and analyse the central mechanisms of acupuncture treatment in patients with SCD. The findings may provide deeper insight into the benefits and mechanisms of acupuncture for patients with SCD.

\section{Trial status}

This trial is currently in the recruitment phase.

\section{Author affiliations}

${ }^{1}$ School of Acupuncture-Moxibustion and Tuina, Beijing University of Chinese Medicine, Beijing, China

${ }^{2}$ Department of Acupuncture and moxibustion, Dongzhimen Hospital Affiliated to Beijing University of Chinese Medicine, Beijing, China

${ }^{3}$ School of Life Sciences, Beijing University of Chinese Medicine, Beijing, China ${ }^{4}$ Beijing Hospital of Traditional Chinese Medicine, Capital Medical University, Beijing, China

Contributors CZL and JWH conceived and designed the experiments. PZ, ZYW, SQH, CQY, GXS, JQL and YNZ performed the experiments. XW, JW and JFT analysed the data. CQY, XW and CZL wrote the paper. All authors approved the final manuscript.

Funding This work was supported by the National Natural Science Foundation of China (Grant No 81674055) and supported by the Fundamental Research Funds for the Central Universities (2017-JYB-JS-018).

Disclaimer We declared the study was only supported by the governmental funding, and the study did not receive funding/assistance from a commercial organisation.

Competing interests None declared.

Patient consent for publication Not required.

Ethics approval Ethics Committee of the Beijing Hospital of Traditional Chinese Medicine Affiliated to Capital Medical University (Ref: 2017BL-061-02).

Provenance and peer review Not commissioned; externally peer reviewed.

Open access This is an open access article distributed in accordance with the Creative Commons Attribution Non Commercial (CC BY-NC 4.0) license, which permits others to distribute, remix, adapt, build upon this work non-commercially, and license their derivative works on different terms, provided the original work is properly cited, appropriate credit is given, any changes made indicated, and the use is non-commercial. See: http://creativecommons.org/licenses/by-nc/4.0/.

ORCID iD

Cun-Zhi Liu http://orcid.org/0000-0001-8031-5667

\section{REFERENCES}

1. Livingston G, Sommerlad A, Orgeta V, et al. Dementia prevention, intervention, and care. The Lancet 2017;390:2673-734.

2. Delbeuck X, Van der Linden M, Collette F. Alzheimer's disease as a disconnection syndrome? Neuropsychol Rev 2003;13:79-92.

3. Ong SS, Doraiswamy PM, Lad EM. Controversies and future directions of ocular biomarkers in Alzheimer disease. JAMA Neurol 2018;75:650.

4. WHO. Dementia: a public health priority. Geneva: World Health Organization-Alzheimer's Disease International hwwimhpdrea.

5. G8 dementia. Summit Declaration. Available: https://www.gov.uk/ government/publications/g8-dementia-summit-agreements

6. Sperling RA, Aisen PS, Beckett LA, et al. Toward defining the preclinical stages of Alzheimer's disease: Recommendations from the National Institute on Aging-Alzheimer's Association workgroups on diagnostic guidelines for Alzheimer's disease. Alzheimer's \& Dementia 2011;7:280-92.

7. Jack CR, Knopman DS, Jagust WJ, et al. Tracking pathophysiological processes in Alzheimer's disease: an updated hypothetical model of dynamic biomarkers. The Lancet Neurology 2013;12:207-16.

8. Villemagne VL, Burnham S, Bourgeat $P$, et al. Amyloid $\beta$ deposition, neurodegeneration, and cognitive decline in sporadic Alzheimer's disease: a prospective cohort study. The Lancet Neurology 2013:12:357-67.

9. Jessen $\mathrm{F}$, Amariglio RE, van Boxtel $\mathrm{M}$, et al. A conceptual framework for research on subjective cognitive decline in preclinical Alzheimer's disease. Alzheimer's \& Dementia 2014;10:844-52.

10. Jonker C, Geerlings MI, Schmand B. Are memory complaints predictive for dementia? A review of clinical and population-based studies. Int J Geriatr Psychiatry 2000;15:983-91.

11. Reisberg B, Gauthier S. Current evidence for subjective cognitive impairment $(\mathrm{SCl})$ as the pre-mild cognitive impairment $(\mathrm{MCl})$ stage of subsequently manifest Alzheimer's disease. Int. Psychogeriatr. 2008;20:1-16.

12. Stewart R. Subjective cognitive impairment. Curr Opin Psychiatry 2012;25:445-50.

13. Scheef L, Spottke A, Daerr M, et al. Glucose metabolism, gray matter structure, and memory decline in subjective memory impairment. Neurology 2012;79:1332-9.

14. van Harten AC, Visser PJ, Pijnenburg YAL, et al. Cerebrospinal fluid $A \beta 42$ is the best predictor of clinical progression in patients with subjective complaints. Alzheimer's \& Dementia 2013;9:481-7.

15. Molinuevo JL, Rabin LA, Amariglio R, et al. Implementation of subjective cognitive decline criteria in research studies. Alzheimers Dement 2017;13:296-311.

16. Rabin LA, Smart CM, Amariglio RE. Subjective cognitive decline in preclinical Alzheimer's disease. Annu Rev Clin Psychol 2017;13:369-96.

17. Smart CM, Karr JE, Areshenkoff CN, et al. Non-Pharmacologic interventions for older adults with subjective cognitive decline: systematic review, meta-analysis, and preliminary recommendations. Neuropsychol Rev 2017;27:245-57.

18. Wang S, Yang H, Zhang J, et al. Efficacy and safety assessment of acupuncture and nimodipine to treat mild cognitive impairment after cerebral infarction: a randomized controlled trial. BMC Complement Altern Med 2016;16:361.

19. Deng M, Wang X-F. Acupuncture for amnestic mild cognitive impairment: a meta-analysis of randomised controlled trials. Acupuncture in Medicine 2016;34:342-8.

20. Jiang DY, HW L. Effect of electroacupuncture on the cognitive function and plasma antibodies against beta-amyloid protein in aged rats with ketamine anesthesia]. Zhongguo Zhong Xi Yi Jie He Za Zhi 2011;31:1502-5.

21. Campanella S. Why it is time to develop the use of cognitive event-related potentials in the treatment of psychiatric diseases. Neuropsychiatr Dis Treat 2013;9:1835-45.

22. de Waal H, Stam CJ, Lansbergen MM, et al. The Effect of Souvenaid on Functional Brain Network Organisation in Patients with Mild 
Alzheimer's Disease: A Randomised Controlled Study. PLoS One 2014;9:e86558.

23. Smart CM, Segalowitz SJ, Mulligan BP, et al. Mindfulness training for older adults with subjective cognitive decline: results from a pilot randomized controlled trial. JAD 2016;52:757-74.

24. Mathotaarachchi S, Pascoal TA, Shin M, et al. Identifying incipient dementia individuals using machine learning and amyloid imaging. Neurobiol Aging 2017;59:80-90.

25. Boutron I, Moher D, Altman DG, et al. Extending the CONSORT statement to randomized trials of nonpharmacologic treatment: explanation and elaboration. Ann Intern Med 2008;148:295-309.

26. Schulz KF, Altman DG, Moher D, et al. Consort 2010 statement: updated guidelines for reporting parallel group randomised trials. International Journal of Surgery 2011;9:672-7.

27. Jia JN ZJ, Xu J, Wei W, et al. The recommendation of diagnosis and treatment of cognitive impairment in Chinese elderly. Chin J Geriatr 2014;33:817-25.

28. Zhao Q, Lv Y, Zhou Y, et al. Short-Term delayed recall of auditory verbal learning test is equivalent to long-term delayed recall for identifying amnestic mild cognitive impairment. PLoS One 2012;7:e51157.

29. Zhao Q, Guo Q, Li F, et al. The shape TRAlL test: application of a new variant of the TRAIL making test. PLoS One 2013;8:e57333.

30. Yang L, Koyanagi A, Smith L, et al. Hand grip strength and cognitive function among elderly cancer survivors. PLoS One 2018;13:e0197909.

31. Cui Z, Zhong S, Xu P, et al. Panda: a pipeline toolbox for analyzing brain diffusion images. Front Hum Neurosci 2013;7:42.

32. Bowie CR, Harvey PD. Administration and interpretation of the TRAIL making test. Nat Protoc 2006;1:2277-81.

33. Chen SP, Bhattacharya J, Pershing S. Association of vision loss with cognition in older adults. JAMA Ophthalmol 2017;135:963-70.

34. Terwindt PW, Hubers AAM, Giltay EJ, et al. Screening for cognitive dysfunction in Huntington's disease with the clock drawing test. Int $J$ Geriatr Psychiatry 2016;31:1013-20.

35. Rivera D, Perrin PB, Stevens LF, et al. Stroop Color-Word interference test: normative data for the Latin American Spanish speaking adult population. NeuroRehabilitation 2015;37:591-624.

36. Moradi E, Hallikainen I, Hänninen T, et al. Rey's auditory verbal learning test scores can be predicted from whole brain MRI in Alzheimer's disease. Neuroimage 2017;13:415-27.

37. Thomas ML, Green MF, Hellemann G, et al. Modeling deficits from early auditory information processing to psychosocial functioning in schizophrenia. JAMA Psychiatry 2017;74:37-46.

38. Rami L, Mollica MA, García-Sanchez C, et al. The subjective cognitive decline questionnaire (SCD-Q): a validation study. JAD 2014;41:453-66.

39. Boccia M, Vecchione F, Piccardi L, et al. Effect of cognitive style on learning and retrieval of navigational environments. Front Pharmacol 2017;8:496.
40. Buysse DJ, Germain A, Moul DE, et al. Efficacy of brief behavioral treatment for chronic insomnia in older adults. Arch Intern Med 2011;171:887-95.

41. Lombardo MV, Auyeung B, Holt RJ, et al. Improving effect size estimation and statistical power with multi-echo fMRI and its impact on understanding the neural systems supporting mentalizing. Neuroimage 2016;142:55-66.

42. Button KS, loannidis JPA, Mokrysz C, et al. Power failure: why small sample size undermines the reliability of neuroscience. Nat Rev Neurosci 2013;14:365-76.

43. Guo Q, Thabane L, Hall G, et al. A systematic review of the reporting of sample size calculations and corresponding data components in observational functional magnetic resonance imaging studies. Neuroimage 2014;86:172-81.

44. Hayasaka S, Peiffer AM, Hugenschmidt CE, et al. Power and sample size calculation for neuroimaging studies by non-central random field theory. Neuroimage 2007;37:721-30.

45. Desmond JE, Glover GH. Estimating sample size in functional MRI (fMRI) neuroimaging studies: statistical power analyses. J Neurosci Methods 2002;118:115-28.

46. Mumford JA, Nichols TE. Power calculation for group fMRI studies accounting for arbitrary design and temporal autocorrelation. Neuroimage 2008;39:261-8

47. Barnes DE, Santos-Modesitt W, Poelke G, et al. The mental activity and eXercise (max) trial: a randomized controlled trial to enhance cognitive function in older adults. JAMA Intern Med 2013;173:797-804

48. Mormino EC, Betensky RA, Hedden T, et al. Amyloid and APOE $\varepsilon 4$ interact to influence short-term decline in preclinical Alzheimer disease. Neurology 2014;82:1760-7.

49. Doraiswamy PM, Sperling RA, Johnson K, et al. Florbetapir F 18 amyloid PET and 36-month cognitive decline:a prospective multicenter study. Mol Psychiatry 2014;19:1044-51.

50. Rabin LA, Smart CM, Crane PK, et al. Subjective cognitive decline in older adults: an overview of self-report measures used across 19 international research studies. JAD 2015;48:S63-S86.

51. Scheltens NME, Galindo-Garre F, Pijnenburg YAL, et al. The identification of cognitive subtypes in Alzheimer's disease dementia using latent class analysis. J Neurol Neurosurg Psychiatry 2016;87:235-43

52. Simon SS, Yokomizo JE, Bottino CMC. Cognitive intervention in amnestic mild cognitive impairment: a systematic review. Neuroscience \& Biobehavioral Reviews 2012;36:1163-78.

53. Tang Y, Zhu Z, Liu Q, et al. The efficacy of cognitive training in patients with vascular cognitive impairment, no dEmentia (the CogVACCINE study): study protocol for a randomized controlled trial. Trials 2016;17:392.

54. Reisberg B, Prichep L, Mosconi L, et al. The pre-mild cognitive impairment, subjective cognitive impairment stage of Alzheimer's disease. Alzheimer's \& Dementia 2008;4:S98-S108. 